\title{
Aproximación terapéutica del uso-abuso de MDMA (Extasis)
}

\author{
Sálz Martínez, P.A.; González García-Portilla, M.; Martínez Barrondo, S.; \\ Bascarán Fernández, M.T.; Bousoño García, M.; Bobes García, J.
}

Area de Psiquiatría - Facultad de Medicina (Universidad de Oviedo)

Enviar correspondencia a:

Pilar Alejandra Sáiz. Area de Psiquiatría - Facultad de Medicina, Julián Clavería 6 - 3³3006 Oviedo

\section{Resumen}

El uso de éstasis (MDMA), una anfetamina sintética, se ha ido popularizando tanto en España como en Europa durante los últimos 10 años, siendo considerada como una sustancia "segura". A pesar de ello, en la literatura científica, se han descrito un no desdeñable número de muertes y reacciones adversas de tipo físico y psíquico relacionadas con su consumo.

El tratamiento del uso-abuso de MDMA está mucho menos desarrollado que el tratamiento de otros tipos de drogas. No obstante, desde la perspectiva del uso-abuso de sustancias, la mejor aproximación terapeútica consistiría en combinar los "tres pilares del tratamiento": biológico, social y psicológico.

Finalmente, se hace referencia al manejo de las reacciones adversas severas, tanto de tipo físico como de tipo psíquico.

Palabras clave: MDMA, tratamiento, uso crónico, reacciones físicas adversas, reacciones psíquicas adversas

\section{INTRODUCCION}

L a MDMA (metilendioximetilanfetamina) es una base sintética derivada de la feniletilamina y relacionada estructuralmente con la sustancia estimulante anfetamina y la sustancia alucinógena mescalina, compartiendo propiedades de ambos compuestos.

Después de unos años de fuerte crecimiento, los niveles de prevalencia del consumo de éxtasis se están estabilizando. La Encuesta Domiciliaria sobre Consumo de Drogas realizada en 1997, pone de manifiesto que esta sustancia ha sido consumida alguna vez por el $2,5 \%$ de los encuestados $(1,0 \%$ de consumidores durante los últimos 12 meses y $0,2 \%$ de consumidores durante el último mes).

No obstante, estas prevalencias se incrementan notablemente cuando se acota el rango de edad. Así, la Encuesta sobre Drogas en Población Escolar (1996), ofrece prevalencias de consumo de éxtasis alguna vez en la vida del $5,1 \%$, consumo en los últimos 12

\section{Summary}

The social use of ecstasy (MDMA), a synthetic amphetamine, has been widespread in Spain and Europe for the last 10 years, and is popularly considered as "safe". However, deaths and serious physical and psychological adverse reactions have occurred.

Despite the relative lack of research in this field, from the perspective of substance abuse, a multi-levelled, individually tailored approach which combines the usual "three pillars of treatment": biological, psychological and social methods, is often the best.

The management of both serious physical and psychological adverse reactions is also discuss.

Key words: MDMA, treatment, chronic abuse, physical adverse reactions, psychological adverse reactions

meses del 3,9\% y consumo en los últimos 30 días del $2,2 \%$; lo que hace que ésta sea una de las sustancias preferidas por nuestros jóvenes tras alcohol, tabaco, cannabis, tranquilizantes y alucinógenos.

Cabe, por último, mencionar que los consumidores puros de éxtasis son prácticamente inexistentes, siendo muy frecuente entre estos sujetos el consumo de otras sustancias como cannabis $(90,9 \%)$, alcohol $(87,2 \%)$, tabaco $(77,3 \%)$, anfetaminas $(41,3 \%)$, cocaína $(40,0 \%)$, opiáceos $(8,9 \%)$ e inhalables $(8,6 \%)$.

\section{DEPENDENCIA Y TOLERANCIA}

La MDMA no puede considerarse una droga adictógena en humanos y no desencadena, al suprimir su administración, un síndrome de abstinencia típico, si bien en tests de autoadministración en animales éstos tienden a administrarse MDMA, lo que concuerda con el uso recreativo de la droga en humanos. 
A pesar de algunos datos discrepantes (Jansen, 1999), los resultados obtenidos en estudios clínicos no permiten concluir que la MDMA tenga el patrón tipo de las drogas dependígenas, ya que no existe en los consumidores ni dependencia física (síndrome de anstinencia), ni dependencia psicológica (deseo compulsivo de consumir la droga).

Existen datos que confirman la tolerancia farmacológica en algunos consumidores, ya que necesitan incrementar la dosis para conseguir los efectos subjetivos iniciales (Greer y Tolbert, 1986; Downing, 1986).

\section{TRATAMIENTO DEL USO-ABUSO DE MDMA}

El tratamiento del uso-abuso de MDMA está actualmente mucho menos desarrollado que el tratamiento de otros tipos de drogas, incluidas las anfetaminas, entre otros motivos por su reciente incorporación como objeto de consumo de masas. Dado que en la literatura científica no existen virtualmente estudios en torno a este tema, los avances realizados en el campo del tratamiento del uso-abuso de otros psicoestimulantes se han hecho en numerosas ocasiones extensibles a esta sustancia. No obstante, desde la perspectiva del uso-abuso de sustancias, la mejor aproximación terapeútica consistiría en combinar los "tres pilares del tratamiento": biológico, social y psicológico (Griffiths y Vingoe, 1997).

\section{Tratamiento farmacológico}

Se trata de un tratamiento totalmente inespecífico y se fundamenta en que la evidencia clínica pone de manifiesto que el uso de determinados fármacos puede ser útil en el tratamiento del uso/abuso de psicoestimulantes, revirtiendo o compensando las neuroadaptaciones a largo plazo producidas por el uso crónico de los mismos. En la tabla 1 se muestran una serie de posibilidades terapeúticas útiles en el trata- miento del uso-abuso de psicoestimulantes, así como los síntomas a los que primordialmente se dirigen y su mecanismo de acción (King y Ellinwood, 1997).

Por otra parte, cabría señalar la existencia de una serie de fármacos que parecen tener cierta capacidad profiláctica de la toxicidad inducida por la MDMA (Colado y Lorenzo, 1995; Colado, 1998). En este sentido habría que destacar los siguientes:

ISRS: Estudios realizados en animales ponen de manifiesto que la fluoxetina tiene cierto efecto preventivo sobre la neurotoxicidad inducida por la MDMA siempre que se administre dentro de las 3 horas anteriores o consecutivas al consumo de MDMA (Schmidt y Taylor, 1987; Schmidt, 1989).

Agentes antioxidantes: Existe cierta evidencia de que los radicales libres pueden estar relacionados con el proceso neurotóxico inducido por la MDMA (Colado y Green, 1995). Por este motivo, algunos usuarios de la MDMA consumen altas dosis de agentes antioxidantes como vitamina $\mathrm{C}$ y $\mathrm{E}$.

Precursores serotoninérgicos: La administración de sustancias como tirosina y triptófano sería útil a la hora de normalizar los niveles de serotonina afectados tras el uso-abuso de la sustancia.

\section{Tratamiento psicológico}

A la hora de plantear la intervención no farmacológica del uso-abuso de MDMA, resulta imprescindible tener en cuenta las características de este tipo de consumo en nuestro medio: policonsumo de fin de semana con características de abuso, escasa repercusión en los centros de atención para drogodependientes y demanda predominantemente de origen familiar en los recursos especializados (Llopis y Paris, 1998).

Las posibilidades de intervención psicológica serían el resultado de complementar terapias de tipo cognitivo con terapias comportamentales y básicamente se centrarían en los aspectos señalados en la tabla 2 (Llopis y Paris, 1998).

\section{Tabla 1. Tratamiento farmacológico del uso-abuso de psicoestimulantes}

\begin{tabular}{|lll|}
\hline Fármaco & Síntomas sobre los que actúa & Mecanismo de acción \\
\hline Agonistas dopaminérgicos & Disforia, anergia & $\uparrow$ actividad DA \\
Antidepresivos tricíclicos & Depresión, craving, disforia & $\uparrow$ tono de activación y afectivo \\
& & $\downarrow$ impulso de consumo \\
ISRS & Elevada impulsividad & $\uparrow$ actividad 5-HT \\
\hline ISRS = Inhibidores Selectivos de Recaptación de Serotonina &
\end{tabular}




\section{Intervención sobre consultantes en fase de consumo}

- Situar a la sustancia y a sus efectos en el contexto real

- Reducir las expectativas del usuario hacia los efectos positivos

- Lograr el acercamiento del paciente para favorecer contactos posteriores

- Informar sobre políticas de reducción de riesgos

\section{Intervención sobre consumidores que han experimentado efectos adversos}

- Situar la sustancia y a sus efectos en el contexto real

- Reducir las expectativas del usuario hacia los efectos positivos

- Lograr un cambio en la relación sustancia - estilo de vida

- Motivar el cese del consumo

\section{Intervención sobre familiares}

- Eliminar actitudes negativas y ambivalentes hacia el consumidor

- Reducir las situaciones de conflicto

- Disminuir respuestas desproporcionadas de la familia

- Enmarcar el problema en su dimensión real

- Fomentar la instauración consensuada de normas de convivencia

- Lograr apoyo familiar al tratamiento

Modificado de Llopis y Paris (1998)

\section{Programas de reducción de daños}

Dado que gran parte de la toxicidad aguda de la MDMA es consecuencia de factores tales como el ambiente donde se consume con elevadas temperaturas, el policonsumo de sustancias, la realización de ejercicio intenso, deshidratación o hiperhidratación, entre otras (Henry, 1992; Newcombe, 1994; Saunders, 1995), en el momento actual se hace gran hincapié, a nivel europeo, en la puesta en marcha de una serie de medidas ambientales y educativas dirigidas a la reducción de daños (EMCDDA, 1997).

En la tabla 3, se resumen una serie de medidas generales de caracter preventivo, que afectan tanto a los locales donde se llevan a cabo eventos que pueden estar relacionados con el consumo de pastillas, como a los propios consumidores.

\section{REACCIONES ADVERSAS ASOCIADAS AL CONSUMO DE MDMA}

El éxtasis ha gozado de una cierta reputación de sustancia "segura", o al menos de bajo para la salud. Esta imagen no deja de tener fundamento ya que, al menos hasta su ilegalización en 1985, el número de reacciones adversas comunicadas (en particular al sistema de notificación estadounidense, DAWN) era muy bajo (Dowling, 1990). No obstante, en Europa esta sustancia ha presentado mayor número y gravedad en sus efectos tóxicos, quizás debido a las diferentes circunstancias en que se consume (Roig, 1994).

Conviene recordar que determinados contextos de consumo como discotecas con elevada temperatura ambiental y poca ventilación, ejercicio físico intenso y mantenido, escasa ingestión de líquidos, etc., podrían contribuir a la aparición de dichos efectos o al agravamiento de los mismos. Por otra parte, se ha producido una reformulación en los años 80 de esta sustancia, no farmacológica sino cultural, al pasar de ser consumida en EE.UU. en círculos muy limitados y relacionados con el movimiento "new age", a ser un producto de mayor difusión e ingerido fundamentalmente en salas de baile como energizante y desinhibidor (Randall, 1992).

Las reacciones adversas asociadas al consumo de MDMA y otras drogas sintéticas tienen un carácter más bien excepcional, resultando llamativa la gran discrepancia existente entre las tasas crecientes de consumos de feniletilaminas y las escasas demandas de asistencia, tanto en centros especializados de drogodependencias como a los profesionales de la salud y/o en los servicios de psiquiatría o de urgencias de los hospitales regionales o comarcales (DGPNSD, 1996).

\section{Reacciones adversas de tipo físico}

En la mayoría de los casos se ha constatado una relación entre la aparición de reacciones adversas y 


\section{Tabla 3. Programas de reducción de daños}

\section{Requisitos dirigidos a los locales:}

- Monitorizar la temperatura y calidad del aire

- Poseer una habitación "tranquila", donde poder descansar

- Proveer agua fresca en baños y bar

- Educar sobre los riesgos del uso de drogas

\section{Los panfletos educativos hacen hincapié en:}

- Vestir ropas ligeras

- Descansar 10 minutos cada hora

- Beber regularmente (no más de 1 litro a la hora), preferentemente zumos

- Mascar chicle

- Comer plátanos

\section{Tabla 4. Dificultades en la atribución causal}

- Baja prevalencia de psicopatología en relación al alto índice de uso/abuso de MDMA

- Alta frecuencia de antecedentes familiares y personales de psicopatología

- Alta frecuencia de psicopatología debida a uso/abuso de otras drogas

- Gran diversidad en lo que se refiere a intensidad / tiempo de consumo de los casos observados

- Alto porcentaje de policonsumo entre los usuarios de MDMA

- Contenido "dudoso" de las pastillas de MDMA

- Relación temporal no bien establecida entre consumo de la sustancia y aparición de la sintomatología

- Ausencia de diferencias fenomenológicas entre las psicosis esquizofrénicas y los trastornos psicóticos relacionados con uso/abuso de MDMA

Tomado de Bobes et al, 1995; 1998.

determinados factores tales como: cierta predisposición previa, consumos excesivos o consumos regulares intensivos. Así, en las personas alérgicas a los derivados anfetamínicos, una pequeña cantidad de MDMA puede dar lugar a la aparición de una reacción anafiláctica de graves consecuencias. Por otra parte, hay que tener presente que no todos los consumos excesivos o regulares se acompañan necesariamente de una reacción adversa aguda (Gamella y Alvárez, 1997).

Hasta la fecha se han descrito básicamente cuatro tipos de reacciones adversas graves, presumiblemente asociadas al consumo de drogas de síntesis: hipertermias malignas, acompañadas de coagulopatías y/o rabdomiolisis e insuficiencia renal aguda, accidentes cerebrovasculares (hemorragias, trombosis e infartos cerebrales), hiponatremias e insuficiencia hepática aguda (para revisión Gamella y Alvárez, 1997).

Hipertermia maligna o "golpe de calor"

La evidencia preclínica sugiere que la temperatura corporal central es una variable importante en la neurotoxicidad inducida por la MDMA. Se sabe que tanto la MDMA, como otros derivados anfetamínicos, elevan la temperatura corporal central. Si la elevación de la temperatura inducida por la MDMA es atenuada o bloqueada (farmacológica o ambientalmente), la neurotoxicidad inducida por la MDMA se atenúa o bloquea. Por el contrario, si se eleva la temperatura corporal central (por aumento de la temperatura ambiente), la potencial neurotoxicidad de la MDMA se incrementa (Farfel y Seiden, 1995).

La relación entre temperatura corporal central y lesión serotoninérgica puede tener una gran importancia en la clínica práctica, sobre todo si se tiene en cuenta su uso en la escena "rave". Los "raves" están usualmente abarrotados de gente y suelen desarrollarse en tórridos almacenes dónde los participantes se entregan a horas y horas de intensa actividad física. Esta combinación de factores probablemente se asocie con una elevación de la temperatura corporal central, incrementando, al menos teóricamente, el potencial neurotóxico de la MDMA (McCann y Ricaurte, 1998).

El resultado clínico de lo expuesto en las líneas anteriores no es otro que la hipertermia maligna o "golpe de calor", caracterizado por una elevación de la temperatura corporal por encima de los $40^{\circ} \mathrm{C}$ y la aparición, de modo más o menos paulatino, de la siguiente sintomatología: fatiga, debilidad, confusión, desorientación, disminución / cese de la sudoración, aparición de calambres musculares, enrojecimiento 
Tabla 5. Tratamiento de los trastornos psiquiátricos asociados al consumo de MDMA

\begin{tabular}{|c|c|c|}
\hline Trastorno & Autor (año) & Tratamiento \\
\hline Trastorno de ansiedad & $\begin{array}{l}\text { McCann y Ricaurte (1992) } \\
\text { Pallanti y Mazzi (1992) } \\
\text { Pallanti y Mazzi (1992) } \\
\text { Pallanti y Mazzi (1992) } \\
\text { McGuire et al (1994) } \\
\text { McGuire et al (1994) } \\
\text { Series et al (1994) } \\
\text { Windhaber et al (1997) }\end{array}$ & $\begin{array}{l}\text { BZD + ADTr (amitriptilina, imipramina) } \\
\text { BZD + IMAOs (tranilcipromina) } \\
\text { ISRSs (fluvoxamina) + ADTr (amitriptilina) } \\
\text { ISRSs (fluvoxamina) } \\
\text { Beta-bloqueantes } \\
\text { ISRSs (fluoxetina) } \\
\text { Lofepramida + psicoterapia } \\
\text { BZD + ISRSs (paroxetina) / ISRSs (paroxetina) }\end{array}$ \\
\hline Depresión & $\begin{array}{l}\text { Benazzi y Mazzoli (1991) } \\
\text { McCann y Ricaurte (1991) } \\
\text { McGuire et al (1994) } \\
\text { Schifano y Magni (1994) } \\
\text { Cohen (1996) }\end{array}$ & $\begin{array}{l}\text { S-adenosil-L-metionina } \\
\text { ISRSs (fluoxetina) } \\
\text { ADTr (amitriptilina) } \\
\text { ISRSs (fluoxetina) } \\
\text { Loracepam + psicoterapia }\end{array}$ \\
\hline Trastornos psicóticos & $\begin{array}{l}\text { McGuire y Fahy (1991) } \\
\text { Creighton et al (1991) } \\
\text { McGuire y Fahy (1991) } \\
\text { Schifano (1991) } \\
\text { Keenan et al (1993) } \\
\text { Wodarz y Böning (1993) } \\
\text { Series et al (1994) } \\
\text { Landabaso et al (1998) }\end{array}$ & $\begin{array}{l}\text { Haloperidol } \\
\text { Trifluoperacina + flupentixol } \\
\text { Sulpiride } \\
\text { Flufenacina + clotiapina + promacina } \\
\text { Clorpromacina } \\
\text { ISRSs } \\
\text { Haloperidol } \\
\text { Olanzapina }\end{array}$ \\
\hline
\end{tabular}

de la piel, respiración superficial, pulso rápido y débil, aparición de convulsiones, agitación y rigidez muscular, pérdida de conocimiento, colapso y finalmente coma y muerte por parada cardiorrespiratoria (Gamella y Alvárez, 1997).

Por otra parte, la gran elevación de la temperatura corporal puede dar lugar a una destrucción masiva del tejido muscular (rabdomiolisis), dado que el músculo se vería obligado a pasar de un metabolismo aerobio a uno anaerobio, debido a la hipoxia existente, lo cual traería como consecuencia la producción de grandes cantidades de ácido láctico que no podrían ser eliminadas por el hígado, produciendo una situación de acidosis metabólica. Los pasos siguientes serían la aparición de convulsiones con la consiguiente liberación de mioglobina, aparición de cuadros de coagulación intravascular diseminada y la posibilidad de un fallo renal agudo que puede llevar a la muerte del individuo (Henry et al, 1992).

Desde el punto de vista terapeútico, existen una serie de acciones inmediatas que pueden ser llevadas a cabo tales como colocar a la persona en un lugar fresco, tratar de disminuir su temperatura corporal mediante la aplicación de paños húmedos, airear al sujeto, administración de una solución de agua y sal, bebida isotónica o simplemente agua si todavía está conciente y proceder a su traslado inmediato a un servicio de urgencias hospitalario (Gamella y Alvárez, 1997).

En lo que al tratamiento hospitalario se refiere, y basándonos en los casos publicados en la literatura, cabría aplicar las siguientes medidas: rehidratación y reducción de la temperatura corporal mediante enfriamiento y ventilación asistida, habiéndose recurrido en ocasiones al uso de dantroleno para ese fin (Mallick y Bodenham, 1997), aunque su eficacia no está totalmente probada. Otras medidas serán forzar una diuresis alcalina para disminuir la acidosis e incluso hemofiltración o hemodiálisis si fuera necesario (Chadwick et al, 1991; Campkin y Davies, 1992; Fahal et al, 1992; Henry et al, 1992; Screaton et al, 1992; Barret y Taylor, 1993; Cregg y Tracey, 1993; Levine et al, 1993; Logan et al, 1993; Watson et al, 1993; Woodrow et al, 1995; Drake y Broadhurst, 1996; Milroy et al, 1996; Mallick y Bodenham, 1997).

\section{Accidentes cerebrovasculares}

Recientes estudios realizados con MDMA en voluntarios humanos (Grob, 1998; Vollenweider et al, 1998; Mas et al, 1999), ponen de manifiesto que la administración en voluntarios humanos de esta sustancia produce incrementos significativos de algunas 
constantes vitales, concretamente de la temperatura corporal, la frecuencia cardíaca y la presión arterial.

Estos efectos suelen remitir a las pocas horas de administración de la sustancia, pero en otras ocasiones pueden llegar a ser fatales. Generalmente se trata de personas con una predisposición previa (presencia de malformación arteriovenosa), en los que el cúmulo de efectos anteriormente descritos pone en marcha un cuadro de coagulación intravascular diseminada, con la aparición de hemorragias (Gamella y Alvárez, 1997).

La sintomatología del cuadro suele incluir intenso dolor de cabeza que puede ir acompañado de vómitos, debilidad muscular o hemiparesia, así como dificultades para hablar, leer, escribir o entender el lenguaje (Gamella y Alvárez, 1997).

La aparición de un accidente cerebrovascular constituye una complicación grave que requiere una hospitalización inmediata del enfermo y su tratamiento es el propio de los accidentes cerebrovasculares: anticoagulación, neurocirugía, rehabilitación, etc., (Harries y De Silva, 1992; Henry et al, 1992; Hughes et al, 1993; Gledhill et al, 1993; Rothwell y Grant, 1993; Manchanda y Connolly, 1993; Logan et al, 1993; Schlaeppi et al, 1999).

\section{Hiponatremia}

Su aparición puede ser debida a dos motivos fundamentales. Por un lado, estaría la excesiva ingesta de agua y líquidos que en muchas ocasiones realiza el usuario de MDMA con fines preventivos (efecto tóxico directo), mientras que en otras ocasiones parece que su aparición está relacionada con una alteración de la secrección de hormona antidiurética (Gamella y Alvárez, 1997).

La sintomatología que más frecuentemente se asocia a estos cuadros es la siguiente: vómitos, convulsiones, agitación, colapso, incontinencia urinaria e incluso estados catatónicos.

La hiponatremia, bien sea dilucional o por alteración de la secrección de hormona antidiurética, requiere el traslado a un servicio de urgencias, produciéndose en la mayoría de los casos una recuperación a los pocos días. Entre las medidas terapeúticas se incluyen administración de tranquilizantes e infusión salina, así como la realización de un TAC cerebral para descartar la posible existencia de edemas cerebrales y proceder a su tratamiento en caso de que existan (Maxwell et al, 1993; Kessel, 1994; Satchell y Connaughton, 1994; Bryden et al, 1995; Lehman, 1995; Demirkiran et al, 1996; Holden y Jackson, 1996; Milroy et al, 1996; Holmes et al, 1999).

\section{Insuficiencia hepática}

En el momento actual la MDMA consituye una de las principales causas de insuficiencia hepática en menores de 25 años (Andreu et al, 1998). En la mayoría de los casos su aparición ha estado relacionada con patrones regulares de mediana o alta intensidad de ingesta de MDMA, aunque también existen descritos casos relacionados con un consumo ocasional o "experimental", motivo por el cual no se puede afirmar rotundamente que la frecuencia e intensidad de consumo de MDMA estén directamente relacionados con la cuantía del daño hepático producido (Gamella y Alvárez, 1997).

Por otra parte, los casos en los que se ha producido una recuperación espontánea tras el cese de la sustancia (y posterior reaparición de síntomas si ésta vuelve a consumirse) hacen pensar en el papel que en algunos de estos casos ha jugado la idiosincrasia personal.

De igual modo, cabe comentar que el mecanismo de producción del daño hepático no está aún claro, no pudiendo afirmarse rotundamente si se debe a un efecto tóxico directo sobre el hígado de la MDMA o es consecuencia de la hipertermia derivada del consumo de la sustancia.

El cuadro suele caracterizarse por la aparición de malestar general, naúseas, vómitos, dolor abdominal, pérdida de apetito, prúrito, letargia, ictericia, orinas colúricas, heces acolúricas, aumento de bilirribuna y transaminasas... Cabe reseñar que en los casos descritos en la literatura se ha descartado que el daño hepático se debiese al uso de drogas por vía intravenosa, a un consumo elevado de alcohol o a agentes infecciosos. Por otra parte, siempre que se ha podido se ha procedido a descartar la existencia de posibles adulterantes con efecto hepatotóxico (Gamella y Alvárez, 1997).

Aunque, como ya se ha comentado, en algunos casos de insuficiencia hepática se ha constatado una recuperación espontánea tras cesar la ingesta de MDMA (Fidler et al, 1996; Roques et al, 1998), la gran mayoría han requerido la realización de transplante parcial o total de hígado (Brown y Osterloh, 1987; Gorard et al, 1992; Henry et al, 1992; Shearman et al, 1992; Ijzermans et al, 1993; Dykhuizen et al, 1995; Huarte y Pueyo, 1995; Khakoo et al, 1995; Skopp et al, 1995; Coore, 1996; Ellis et al, 1996; Brauer et al, 1997).

\section{Reacciones adversas de tipo psíquico}

Hasta la fecha se han descrito relativamente pocos trastornos neuropsiquiátricos relacionados con el uso de MDMA, sobre todo si se tiene en cuenta la elevada prevalencia de trastornos psicopatológicos previos en consumidores de sustancias psicoactivas, encontrada por numerosos autores.

No obstante, desde el comienzo de los 90 está siendo cada vez más frecuente la aparición de publicaciones referentes a complicaciones psiquiátricas asociadas al uso de esta sustancia, aunque, en líneas generales, es difícil precisar si ésta interviene como 
factor facilitador, desencadenante o es capaz de producir dichos trastornos "ex novo", ya que la revisión de los casos pone de manifiesto la existencia de una elevada tasa de policonsumo de drogas en estos pacientes, así como la existencia de trastornos psiquiátricos previos y / o morbilidad psiquiátrica entre familiares (tabla 4).

Por otra parte, el tiempo de consumo de MDMA y la duración del mismo no constituyen parámetros útiles a la hora de predecir si van a existir o no complicaciones psiquiátricas (Wodarz y Böning, 1993), ya que una sola o muy escasas dosis se han asociado con la aparición de psicosis paranoide u otros trastornos (Williams et al, 1993).

Conviene además recordar que en los años en que fue utilizada en contextos terapeúticos no fue descrito ningún trastorno asociado a su uso (Roig, 1993), y el hecho de que, en muchas ocasiones, se consume junto a otras sustancias, y que, como ya hemos visto, suele estar adulterada en proporciones importantes.

Las manifestaciones psiquiátricas más frecuentemente asociadas al consumo de MDMA pueden agruparse en cinco grandes grupos: trastornos de ansiedad, depresión, trastornos psicóticos, "flashbacks" y/o alucinaciones y otras alteraciones (Bobes et al, 1998). El tratamiento de dichos trastornos no difiere, en líneas generales, del tratamiento que se realizaría independientemente de que la MDMA fuera la causante o no del cuadro (tabla 5).

\section{CONCLUSIONES}

En el momento actual no disponemos de un tratamiento específico para el uso/abuso de MDMA

Cada vez existen más evidencias que ponen de manifiesto la eficacia preventiva de los programas de reducción de daños

La investigación básica pone de manifiesto la posible utilidad profiláctica de determinadas sustancias (ISRSs, sustancias antioxidantes, precursores serotoninérgicos)

Las reacciones adversas de índole física / psíquica se tratan sintomáticamente en función del trastorno existente

\section{BIBLIOGRAFIA}

Andreu V, Mas A, Bruguera M, Salmerón JM, Moreno V, Nogué $S$ et al (1998): Ecstasy: a common cause of severe acute hepatotoxicity. J Hepatol, 29: 394-397.
Barret PJ, Taylor GT (1993): Ecstasy ingestion: a case report of severe complications. Journal of the Royal Society of Medicine, 86: 233-234.

Benazzi F, Mazzoli M (1991): Psychiatric illness associated with "ecstasy". Lancet, 338: 1520.

Bobes J (1995): Extasis: Aspectos farmacológicos, psiquiátricos y médico-legales. Barcelona: Ediciones Neurociencias.

Bobes J, González MP, Sáiz PA, González JC (1998): Psicopatología y trastornos psiquiátricos relacionados con el uso de MDMA. En: Bobes J, Lorenzo P, Sáiz PA eds. Extasis (MDMA): un abordaje comprehensivo. Barcelona: Masson SA. p. 173-197.

Brauer RB, Heidecke CD, Nathrath W, Beckurts KT, Vorwald P, Zilker TR et al (1997): Liver transplantation for the treatment of fulminant hepatic failure induced by the ingestion of ecstasy. Transpl Int, 10: 229-233.

Brown C, Osterloh J (1987): Multiple severe complications from recreational ingestion of MDMA ("ecstasy"). JAMA, 258: 780-781.

Bryden AA, Rothwell PJN, Oreilly PH (1995): Urinary retention with misuse of ecstasy. BMJ, 310: 504.

Campkin NTA, Davies UM (1992): Another death from ecstasy. Journal of the Royal Society of Medicine, 85: 61.

Chadwick IS, Curry PD, Linsley A, Freemont AJ, Doran B (1991): Ecstasy, 3-4 methylenedioxymethamphetamine (MDMA), a fatality associated with coagulopathy and hyperthermia. Journal of the Royal Society of Medicine, 84: 371.

Cohen RS (1996): Adverse symptomatology and suicide associated with the use of methylenedioxymethamphetamine (MDMA; “Ecstasy"). Biol Psychiatry, 39: 819-820.

Colado MI, Green AR (1995): The spin trap reagent alphaphenyl-N-tert-butylnitrone prevents "ecstasy" -induced neurodegeneration of 5-hydroxytryptamine neurones. Eur J Pharmacol, 280: 343.

Colado MI, Lorenzo P: MDMA (éxtasis) (1995): Farmacología y toxicología. En: Bobes J, ed. Extasis: Aspectos farmacológicos, psiquiátricos y médico-legales. Barcelona: Ediciones Neurociencias. p. 1-46.

Colado MI (1998): Neurotoxicidad inducida por MDMA y su prevención farmacológica. En: Bobes J, Lorenzo P, Sáiz PA, eds. Extasis: Un abordaje comprehensivo. Barcelona: Masson, SA. p. 41-72.

Coore JR (1996): A fatal trip with ecstasy: a case of 3,4 methylenedioxymethamphetamine / 3,4 methylenedioxyamphetamine toxicity. Journal of the Royal Society of Medicine, 89: 51P-52P.

Cregg MT, Tracey JA (1993): Ecstasy abuse in Ireland. Irish Medical Journal, 86: 118-120.

Creighton FJ, Black DL, Hyde CE (1991): Ecstasy psychosis and flashbacks. BR J Psychiatry, 159: 713-715.

DGPNSD (1996): Memoria 1995. Madrid: Ministerio de Justicia e Interior.

DGPNSD (1997): Encuesta sobre drogas a la población escolar, 1996. Madrid: Ministerio de Justicia e Interior. 
DGPNSD (1998): Encuesta domiciliaria sobre consumo de drogas, 1997. Madrid: Ministerio de Justicia e Interior.

Demirkiran M, Jankovic J, Dean JM (1996): Ecstasy intoxication: an overlap between serotonin syndrome and neuroleptic malignant syndrome: Clin Neuropharmacol, 19: 157-164.

Dowling GP (1990): Human deaths and toxic reactions attributed to MDMA and MDEA. En: Peroutka SJ ed. Ecstasy: the clinical, pharmacological and neurotoxicological effects of the drug MDMA. Hingham, Mass: Kluwer Academic Publishers.

Downing J (1986): The psychological and physiological effects of MDMA on normal volunteers. J Psychoactive Drugs, 18: 335-340.

Drake WM, Broadhurst PA (1996): QT-interval prolongation with ecstasy. South African Medical Journal, 86: 180181.

Dykhuizen RS, Brunt PW, Atkinson P, Simpson JG, Smith CC (1995): Ecstasy induced hepatitis mimicking viral hepatitis. Gut, 36: 939-941.

Ellis AJ, Wendon JA, Portmann P, Williams R (1996): Acute liver damage and ecstasy ingestion. Gut, 38: 454-458.

EMCDDA (1997): New trends in synthetic drugs in the European Union. Portugal: EMCDDA.

Fahal IH, Sallomi DF, Yaqoob M, Bell GM (1992): Acute-renalfailure after ecstasy. BMJ, 305: 29.

Farfel GM, Seiden LS (1995): Role of hypothermia in the mechanism of protection against serotonergic toxicity. I. Experiments using 3,4-methylenedioxymethamphetamine, dizocilpine, CGS 19755 and NBOX. J Pharmacol Exp Ther, 272: 860.

Fidler H, Dhillon A, Gertner D, Burroughs A (1996): Chronic ecstasy (3,4-methylenedioxymethamphetamine) abuse: a recurrent and unpredictable cause of severe acute hepatitis. J Hepatol, 25: 563-566.

Gamella JF, Alvárez A (1997): Drogas de síntesis en España. Patrones y tendencias de adquisición y consumo. Madrid: DGPNSD.

Gledhill JA, Moore DF, Bell D, Henry JA (1993): Subarachnoid haemorrhage associated with MDMA abuse. Journal of Neurology Neurosurgery and Psychiatry, 56: 1036-1037.

Gorard DA, Davies SE, Clark ML (1992): Misuse of ecstasy. BMJ, 305: 309.

Greer G, Tolbert P (1986): Subjective reports of the effects of MDMA in a clinical setting. J Psychoactive Drugs, 18: 319-327.

Griffiths P, Vingoe L (1997): The use of amphetamines, ecstasy and LSD in the European Community: A review of data on comsumption patterns and current epidemiological literature. Report prepared for the EMCDDA by the National Addiction Centre, London. London: National Addiction Centre.

Grob Ch (1998): Investigación humana con MDMA. En: Bobes J, Lorenzo P, Sáiz PA eds. Extasis (MDMA): un abordaje comprehensivo. Barcelona: Masson SA. p. 99105.
Harries DP, De Silva R (1992): "Ecstasy" and intracerebral haemorrhage. Scottish Medical Journal, 37: 150-152.

Henry JA (1992): Ecstasy and the dance of death. BMJ, 305: 5-6.

Henry JA, Jeffreys KJ, Dawling S (1992): Toxicity and deaths from 3,4-methylenedioxymethamphetamine ("ecstasy"). Lancet, 340: 384-387.

Holden R, Jackson MA (1996): Near-fatal hyponatraemic coma due to vasopressin oversecretion after "ecstasy" (3,4-MDMA). Lancet, 347: 1052.

Holmes SB, Banerjee AK, Alexander WD (1999): Hyponatraemia and seizures after ecstasy use. Postgrad Med J, 75: 32-33.

Huarte MP, Pueyo AM (1995): Hepatitis aguda a causa de la ingestión de éxtasis. Revista Española de Enfermedades Digestivas, 87: 681-683.

Hughes JC, McCabe MC, Evans RJ (1993): Intracranial haemorrhage associated with ingestion of "ecstasy". Source Archives of Emergency Medicine, 10: 372-374.

ljzermans JNM, Tilanus HW, De Mann RA, Metselaar HJ (1993): Ecstasy and liver transplantation. Annales de Medicine Interne, 144: 568.

Jansen KL (1999): Ecstasy (MDMA) dependence. Drug Alcohol Depend, 53: 121-124.

Kessel B (1993): Hyponatraemia after ingestion of ecstasy. BMJ, 308: 414.

Khakoo SI, Coles CJ, Armstrong JS, Barry RE (1995): Hepatotoxicity and accelerated fibrosis following 3,4-methylenedioxymethamphetamine ("ecstasy"). J Clin Gastroenterol, 20: 244-247.

Keenan E, Gervin M, Dorman A, O'Connor JJ (1993): Psychosis and recreational use of MDMA ("ecstasy"). Irish J Psychol Medicine, 10: 162-163.

King GR, Ellinwood Jr EH (1997): Amphetamines and other stimulants. En: Lowinson JH, Ruiz P, Millman RB, Langrod JG, eds. Substance abuse. A comprehensive textbook. 3th edition. Baltimore: Williams \& Wilkins. p. 207-223.

Landabaso MA, Sanz J, Calle R, Iraurgi I, Ruiz de Apodaka J (1998): Utilidad clínica de la olanzapina en el abuso / adicción de drogas de síntesis (MDMA). Actas Luso Esp Neurol Psiquiatr Cienc Afines, 26 (número especial): 107.

Lehman E, Thom CH, Croft DN (1995): Delayed severe rhabdomyolysis after taking ecstasy. Postgrad Med J, 71: 186-187.

Levine AJ, Drew S, Rees GM (1993): Ecstasy induced pneumomediastinum. Journal of the Royal Society of Medicine, 86: 232-233.

Llopis JJ, Paris B (1998): Servicios de atención y orientación telefónica a familiares y consumidores de MDMA. En: Bobes J, Lorenzo P, Sáiz PA, eds. Extasis: Un abordaje comprehensivo. Barcelona: Masson, SA. p. 199-211.

Logan A, Stickle B, O'Keefe N, Hewitson H (1993): Survival following "ecstasy" ingestion with a peak temperature of 42 degrees centigrade. Anaesthesia, 48: 1017-1018. 
Mallick A, Bodenham AR (1997): MDMA induced hyperthermia: a survivor with an initial body temperature of 42.9 degrees C. J Accid Emerg Med, 14: 336-338.

Manchanda S, Connolly MJ (1993): Cerebral infarction in association with ecstasy abuse. Postgraduate Medical Journal, 69: 874-889.

Mas M, Farré M, de la Torres R, Roset PN, Ortuño J, Segura $J$ et al (1999): Cardiovascular and neuroendocrine effects and pharmacokinetics of 3,4-methylenedioxymethamphetamine in humans. J Pharmacol Exp Ther, 290: 136-145.

Maxwell DL, Polkey MI, Henry JA (1993): Hyponatremia and catatonic stupor after taking ecstasy. BMJ, 307: 1399.

McCann UD, Ricaurte GA (1991): Lasting neuropsychiatric sequelae of methylenedioxymethamphetamine ("ecstasy") in recreational uses. J Clinical Psychopharm, 11: 302-305.

McCann UD, Ricaurte GA (1992): MDMA ("ecstasy") and panic disorder. Biol Psychiatry, 32: 950-953.

McCann UD, Ricaurte GA (1998): Aproximación a la neurobiología y neurotoxicidad comparada inducida por la MDMA. En: Bobes J, Lorenzo P, Sáiz PA eds. Extasis (MDMA): un abordaje comprehensivo. Barcelona: Masson SA. p. 89-97.

McGuire P, Fahy T (1991): chronic paranoid psychosis after misuse of MDMA ("ecstasy"). BMJ, 302: 697.

McGuire PK, Cope H, Fahy TA (1994): Diversity of psychopathology associated with use of 3,4-methylenedioxymethamphetamine ("ecstasy"). British J Psychiatry, 165: 391-395.

Milroy CM, Clark JC, Forrest ARW (1996): Pathology of death associated with "ecstasy" and "eve" misuse. Journal of Clinical Pathology, 49: 149-153.

Newcombe R (1994): Guidelines for good practice at dance parties and nightclubs. Liverpool: 3D Research Bureau.

Pallanti S, Mazzi D (1992): MDMA (ecstasy) precipitation of panic disorders. Biol Psych, 32: 91-94.

Randall T (1992): Ecstasy-fueled "rave" parties become dances of death for english youths. JAMA, 268: 15051506.

Roig A (1993): Efectos adversos asociados al uso recreativo de éxtasis. En: IV Congreso de la Sociedad Española de Toxicomanías. Valencia.

Roig Traver A (1994): Sobre el uso recreativo de la metilendioximetanfetamina: aspectos históricos y efectos adversos. Adicciones, 6: 437-452.

Roques V, Perney P, Beaufort P, Hanslik B, Ramos J, Durand $L$ et al (1998): Acute hepatitis due to ecstasy. Presse Med, 27: 468-470.

Rothwell PM, Grant R (1993): Crerebral venous sinus thrombosis induced by ecstasy. Journal of Neurology Neurosurgery and Psychiatry, 56: 1035.

Satchell SC, Connaughton M (1994): Inappropiate antidiuretic-hormone secretion and extreme rises in serum creatinine kinase following MDMA ingestion. British Journal of Hospital Medicine, 51: 495.

Saunders N (1995): Ecstasy and the dance culture. London: Neal's Yard Desktop Publishing Studio.
Schifano F (1991): Chronic atypical psychosis associated with MDMA ("ecstasy") abuse. Lancet, 338: 1335.

Schifano F, Magni G (1994): MDMA ("ecstasy") abuse: psychopathological features and craving for chocolate: a case series. Biol Psychiatry, 36: 763-767.

Schlaeppi M, Prica A, de Torrente A (1999): Cerebral hemorrhage and "ecstasy". Schweiz Rundsch Med Prax, 88: 568-572.

Schmidt CJ, Taylor VL (1987): Acute effects of methylenedioxymethamphetamine (MDMA) on 5-HT synthesis in the brain. Pharmacologist, 29: Abs. 224.

Schmidt CJ (1989): Acute anf long-term neurochemical effects of methylenedioxymethamphetamine in the rat. En: Peroutka SD, ed. Ecstasy: the clinical, pharmacological and neurotoxiocological effects of the drug MDMA. Massachussets: Kluwer Academic Publishers.

Screaton GR, Cairns HS, Sarner M, Singer M, Thrasher A, Cohen SL (1992): Hyperpyrexia and rhabdomyolysis after MDMA (ecstasy) abuse. Lancet, 339: 677-678.

Series H, Boeles S, Dorkins E, Peveler R (1994): Psychiatric complications of ecstasy use. J Psychopharmacol, 8: 60-61.

Shearman JD, Satsangi J, Chapman RWG, Ryley NG, Weatherhead S (1992): Misuse of ecstasy. BMJ, 305: 309.

Skopp G, Aderjan R, Koster J (1995): Hair analysis in the diagnosis of toxic hepatitis after drug abuse. Dentsche Medizinische Wochenschrift, 120: 1165-1168.

Vollenweider FX, Gamma A, Lietchi M, Huber T (1998): Psychological and cardiovascular effects and short-term sequelae of MDMA ("ecstasy") in MDMA-naive healthy volunteers. Neuropsychopharmacology, 19: 241-251.

Watson JD, Ferguson C, Hinds CJ, Skinner R, Coakley JH (1993): Exertional heat stroke induced by amphetamine analogues. Does dantrolene have a place?. Anaesthesia, 48: 1057-1060.

Williams H, Meagher D, Galligan P (1993): MDMA ("ecstasy"); a case of possible drug-induced psychosis. Ir J Med Sci, 162: 43-44.

Windhaber J, Maierhofer D, Dantendorfer K (1997): Panic disorder induced by methylenedioxymethamphetamine resolved by paroxetine. European Neuropsychopharmacology, 7 (Suppl. 2): S240.

Wodarz N, Boning J (1993): "Ecstasy" induced psychotic despersonalization syndrome. Nervenarzt, 64: 478480.

Woodrow G, Harnden P, Turney JH: Acute renal failure due to accelerated hypertension following ingestion of 3,4methylenedioxymethamphetamine ("ecstasy"). Nephrology Dialysis Transplantation 1995; 10: 399-400. 
